\title{
BMJ Open Venous thromboembolism in medical patients during hospitalisation and 3 months after hospitalisation: a prospective observational study
}

\author{
Alhossain A Khalafallah, ${ }^{1,2,3}$ Brooke E Kirkby, ${ }^{4}$ Sophia Wong, ${ }^{1}$ Yi Chao Foong, ${ }^{1}$ \\ Nishant Ranjan, ${ }^{1}$ James Luttrell, ${ }^{1}$ Ronnie Mathew, ${ }^{2}$ Charles M Chilvers, ${ }^{2}$ \\ Emily Mauldon, ${ }^{2}$ Colin Sharp, ${ }^{1}$ Terry Hannan ${ }^{1,3}$
}

To cite: Khalafallah $A A$, Kirkby BE, Wong S, et al. Venous thromboembolism in medical patients during hospitalisation and 3 months after hospitalisation: a prospective observational study. BMJ Open 2016;6: e012346. doi:10.1136/ bmjopen-2016-012346

- Prepublication history for this paper is available online. To view these files please visit the journal online (http://dx.doi.org/10.1136/ bmjopen-2016-012346).

Received 21 April 2016 Revised 11 July 2016 Accepted 14 July 2016

\section{CrossMark}

\author{
${ }^{1}$ Department of Medicine, \\ Launceston General Hospital, \\ Launceston, Tasmania, \\ Australia \\ ${ }^{2}$ School of Health Sciences \\ and School of Medicine, \\ University of Tasmania, \\ Launceston, Tasmania, \\ Australia \\ ${ }^{3}$ Menzies Institute for Medical \\ Research, University of \\ Tasmania, Australia \\ ${ }^{4}$ Pathology Department, \\ Launceston General Hospital, \\ Launceston, Tasmania, \\ Australia
}

Correspondence to Professor Alhossain Khalafallah;

khalafallah@dhhs.tas.gov.au

\section{ABSTRACT}

Objectives: This study was conducted to assess the incidence and risk factors for venous

thromboembolism (VTE) in a cohort of medical patients both during the period of hospitalisation and following discharge.

Design: This was a prospective observational study to document the risk profile and incidence of VTE posthospitalisation among all medical patients admitted to our institution during the trial period.

Settings: Primary healthcare. Single tertiary referral centre, Tasmania, Australia.

Participants: A total of 986 patients admitted to the medical ward between January 2012 and September 2012 were included in the study with male to female ratio of 497:489. The mean age of patients was 68 years (range 17-112, SD 16).

Results: Overall, $54 / 986$ patients (5.5\%) had a VTE during the study period. Of these, $40 / 54(74.1 \%)$ occurred during hospitalisation and 14/54 (25.9\%) occurred following discharge. VTE risk factors revealed in multivariate analysis to be associated with a previous diagnosis of VTE $(p<0.001,0 R=6.63,95 \% \mathrm{Cl}$ 3.3 to 13.36), the occurrence of surgery within the past 30 days $(\mathrm{p}<0.001,0 \mathrm{R}=2.52,95 \% \mathrm{Cl} 1.33$ to 4.79 ) and an admission diagnosis of pulmonary disease ( $p<0.01$, OR $3.61,95 \% \mathrm{Cl} 1.49$ to 8.76 ). Mobility within 24 hours of admission was not associated with an increased risk. There was risk of VTE when the length of stay prolonged $(p=0.046$, $\mathrm{OR}=1.01,95 \% \mathrm{Cl} 1.00$ to 1.03 ), however it was not sustained with multivariate modelling. VTE-specific prophylaxis was used in $53 \%$ of the studied patients. Anticoagulation including antiplatelet agents were administered in $63 \%$ of patients who developed VTE.

Conclusions: This prospective observational study found that $5.5 \%$ of the studied patients developed VTE. Among those, 25.9\% (14/54) of patients had a detected VTE posthospitalisation with this risk being increased if there was a history of VTE, recent surgery and pulmonary conditions. Thromboprophylaxis may be worth considering in these cohorts. Further study to confirm these findings are warranted.

Trial registration number: ACTRN12611001255976.

\section{Strengths and limitations of this study}

- Venous thromboembolism (VTE) is one of most common disease that affects inpatients, and in the majority of cases it is preventable.

- Closing the loop between medical admission to hospital and after discharge will be a decisive factor in improving the prevention strategy for VTE in timely fashion, and to reduce morbidity and mortality associated with VTE and improve outcomes.

- There is limited data regarding number of medical patients who develop VTE after their medical admission. Therefore, it is essential to study the risk factors involved with formation of VTE and at the same time develop a prevention strategy.

- We found $5.4 \%$ of the studied patients developed VTE. Among those $25.9 \%$ had a detected VTE posthospitalisation with this risk being increased if there was a history of VTE, recent surgery and pulmonary conditions.

- Our study was limited to a single centre with a relatively small number of patients and there were no investigations performed routinely to detect asymptomatic VTE, during or after discharge.

\section{INTRODUCTION}

Venous thromboembolism (VTE), defined as deep venous thrombosis (DVT) and pulmonary embolism (PE), has traditionally been seen as a complication of major surgery. However, this view has been challenged by a number of recent studies demonstrating that both surgical and acute medical patients have an elevated risk of developing VTE. ${ }^{1}$ Furthermore, while hospitalised patients were over 100 times more likely to develop VTE compared with the rest of the community, a significant number of cases also developed VTE subsequent to discharge. ${ }^{23}$ 
There has been increasing emphasis on VTE prevention as PE is one of the most common causes of death in hospital, accounting for $10-30 \%$ of hospital deaths. ${ }^{34}$ In absolute numbers, this accounts for 35387 VTE-related hospital separations in Australia from 2007 to 2008 and 2000 deaths each year. ${ }^{5}$ The incidence of VTE ranges from 2.39 per 1000 separations in the USA to 4.5 per 1000 separations in Australia. When broken down into DVT and PE, these occurred at a rate of 1.52 and 1.21 per 1000 hospitalisations in the USA to 2.3 and 2.2 per 1000 separations in Australia during a similar period, respectively. ${ }^{5}$ An estimated $20-25 \%$ of PE cases present as sudden death. The majority of these deaths could be prevented by providing adequate appropriate prophylaxis for patients identified as at-risk individuals. $^{37}$

In addition to the adverse patient outcomes of VTE, it also represents a significant financial burden on the health budget. An inpatient VTE incident study analysing 2147 patients found that the median cost of VTE events was US $\$ 3131$ per DVT, US $\$ 6424$ per PE and US $\$ 6678$ per DVT+PE event. ${ }^{8}$ Other studies have reported a similar substantial cost burden related to VTE and its consequences. $^{9-12}$ In Australia and other developed nations, the most commonly used pharmacological agents include low molecular weight heparin and unfractionated heparin sodium. Others include aspirin, warfarin, dabigatran etexilate, rivaroxiban, fondaparinux and danaparoid. Mechanical options include graduated compression stockings and pneumatic venous pump devices that intermittently compress muscles in the lower limbs. ${ }^{5} 13$

Current guidelines in Australia, as per the National Health and Medical Research Council (NHMRC), recommend formal assessment of VTE risk for all patients admitted to hospital together with a decision about prophylaxis, taking into account relevant contraindications to pharmacological and mechanical prophylaxis. ${ }^{14}$

Patients considered to be at high risk of developing VTE who should be considered for prophylaxis are those who have had major surgery, trauma, certain fractures and immobility or paralysis. ${ }^{15}$ Additional risk factors that are not necessarily significant enough in isolation to warrant prophylaxis include previous VTE, increasing age, cardiac or respiratory failure, oestrogens, varicose veins, pregnancy and the puerperium, malignant neoplasm, chemotherapy, central venous catheter and some haematological conditions. ${ }^{15}{ }^{16}$ Physicians should consider predisposing risks, or a combination thereof, in conjunction with the clinical situation to determine the appropriate type and duration of VTE prophylaxis, keeping in mind that the predictive values of VTE events are different for all factors. ${ }^{17}$

While it is generally agreed that VTE in medical patients is a significant issue and prophylactic measures are effective at reducing the risk, the implementation of VTE prophylaxis in this group remains low, at around $40 \%,{ }^{18}$ and rates of DVT, PE and VTE as a whole have not decreased in the past decade. ${ }^{5}{ }^{19}$ Contributing to this may be the lack of consensus regarding individual patient's risk assessment, including the relative weight of the various risk factors, application of contraindications and the risk-benefit profile of different prophylactic measures available.

There have been limited real-life patient cohort studies that have examined the incidence and risk factors of VTE in medical patients and much of the current understanding on the topic comes from the study of patient subgroups within randomised controlled trials (RCTs) rather than from real-life cohorts. ${ }^{20}$ We found that there are even fewer studies examining the postdischarge period.

\section{Aims of the study}

This study examined VTE (DVT and PE) incidence and risk factors in a cohort of medical patients in a mediumsized tertiary referral hospital, both during the period of hospitalisation and following discharge. It is hoped that this study will help further our understanding of VTE in this population group and shape future guideline development.

\section{METHODS}

This cohort study aimed to document the risk profile and incidence of VTE among all medical patients admitted to the Launceston General Hospital (LGH), Tasmania, Australia, during the trial period from January to September 2012. The trial was registered prospectively in the Australian New Zealand Clinical Trials Registry (https://www.anzctr.org.au/Trial/Registration/ TrialReview.aspx?ACTRN=12611001255976).

All consecutive patients admitted to a medical ward during the study period were eligible for inclusion $(n=1478)$, however with short admissions $(27 \%)$ and idiosyncratic medical record keeping, only 986 participants $(66.6 \%)$ were included in the analysis. As the primary data sources were routine hospital records, participant information was not complete across all data fields.

Any diagnosis of a VTE (DVT or PE) event (including death) up to 3 months from the indexed admission was assessed. AVTE event was documented if:

A. A diagnosis code of VTE appeared in a hospital record (in the indexed admission or a posthospitalisation or emergency admission in any Tasmanian hospital);

B. A diagnosis of VTE as confirmed via Doppler ultrasound, CT pulmonary angiography, ventilation/ perfusion scan or coagulation tests existed in addition to the hospital's imaging and pathology records;

C. A cause of death related to VTE appeared in the death registry data.

Patients were evaluated prospectively for all VTE risk factors (including age, sex, weight, different organs function, mobility, medications and other laboratory risk 
factors) on admission and submitted electronically after admission together with a record of all relevant comorbidities. The patients were monitored for 3 months following discharge to assess the occurrence of VTE. Patients with full anticoagulation regimen for different indications such as valve replacement, atrial fibrillation and long-term anticoagulation were not included in the univariate analysis despite being part of the study. There was no VTE recorded in this cohort.

\section{Setting and participants}

This observational study was conducted at the LGH, a tertiary hospital servicing a rural and regional population in Northern Tasmania. Patients were admitted to the LGH between 17 January 2012 and 6 September 2012. A total of 986 patients with sufficiently detailed medical records were included in the study, $50.5 \%$ of whom were male $(n=497)$ and $49.5 \%$ of whom were female $(n=489)$. Total number of admitted patients during the trial period was 1478 patients. Patients excluded from the study were those with admission $<48$ hours and patients who were not contactable.

As the study was exploratory, data were collected on a broad range of risk factors for which there was a plausible clinical association with the occurrence of VTE. Age, weight, height, body mass index (BMI, calculated as weight in kilograms divided by the square of height in metres) and smoking status were assessed. Relevant medical history included previously diagnosed VTE, surgery or trauma resulting in a fracture within the past 30 days. Diagnoses of diabetes mellitus, hyperlipidaemia, hypertension, varicose veins, acute non-infectious inflammatory disease, heart failure and active malignancy were considered as relevant comorbidities. Factors pertaining to the current admission included estimated glomerular filtration (eGFR) and creatinine clearance levels, medications relevant to VTE, bleeding contraindications, acute infectious disease, DVT prophylaxis, use of a vascular access device, mobility within 24 hours of admission and length of stay (LOS).

BMI was considered as a categorical variable with four and eight categories (according to the WHO classifications); however, no statistically significant association between VTE and BMI was present. A statistically significant association was present for height and weight when treated as a continuous variable, but when a number of classification schemes were used to categorise height and weight, this association could not be replicated. Other continuous variables were preadmission eGFR and eGFR at admission, creatinine clearance and LOS. Dichotomous measures included smoking status, previous VTE, presence of hyperlipidaemia, hypertension, varicose veins, acute infectious disease, acute noninfectious inflammatory disease, and recent surgery or fracture trauma (within the past 30 days). A free-text field noting other risk factors relevant to VTE was originally included and was manually recoded as a dichotomous variable and no statistically significant association was found.

Admission and discharge diagnoses were recorded and extracted from the hospital records. Data contained a combination of International Classification of Diseases (ICD) clinical diagnoses and free-text comments. Following data cleaning and preliminary analyses diagnosis was coded within the five categories listed in table 1 (cardiology, pulmonary, oncology/haematology, surgery and other).

Patients with the initial diagnosis of VTE at the time of presentation were generally treated with the new oral anticoagulation therapy as outpatients, rather than admitted to our institution. As a result, these patients were automatically not included in the trial without any influence of the study design.

\section{Category of diagnosis}

'Cardiology' included patients admitted with ST segment elevation myocardial infarction (STEMI), non-STEMI, heart failure, hypertension and cardiac arrhythmias. 'Pulmonary' included patients admitted mainly with chronic obstructive pulmonary disease (COPD), pneumonia or pneumothorax. 'Oncology/ haematology' included patients admitted with haematology or oncological malignant diseases. Although we studied medical patients only, the category of 'surgery' group refers to medical patients with any surgical intervention within the past 30 days regardless of their current admission diagnosis. 'Other' diagnoses included gastroenterology, neurology, infection, cellulitis, dehydration and general medical admissions.

\section{Data sources/measurement}

Data on potential VTE risk factors were extracted from the patient records and the type of admission was determined using relevant current clinical guidelines. VTE prophylaxis data were obtained from the patient's medical charts. Record extraction was validated using interdata reliability for random samples of cases.

Patients were evaluated prospectively for all VTE risk factors (including age, sex, weight, different organs function, mobility, medications and other laboratory risk factors) on admission. Data extracted for the study included a record of all relevant comorbidities according to the medical records.

Any occurrence of a VTE (DVT or PE) event (including death) up to 3 months from the indexed admission was assessed. A VTE event was considered to have occurred if a diagnosis code of VTE appeared in the hospital record (in the indexed admission, posthospitalisation admission or emergency department attendance in any hospital), a diagnosis of VTE, confirmed via Doppler ultrasound, CT pulmonary angiography, ventilation/perfusion scan or coagulation tests, existed in the hospital's imaging and pathology records, or a cause of death related to VTE appeared in death registry data. As this was an observational, non-interventional study, there 
Table 1 Univariate association of risk factors with venous thromboembolism in medical patients

\begin{tabular}{|c|c|c|c|c|}
\hline & Number of patients (\%) & Crude OR & $95 \% \mathrm{Cl}$ & p Value \\
\hline \multicolumn{5}{|l|}{ Gender } \\
\hline Male & 497/986 (50.4) & (reference) & & \\
\hline Female & 489/986 (49.6) & 0.74 & 0.42 to 1.28 & 0.28 \\
\hline \multicolumn{5}{|l|}{ Age } \\
\hline$<60$ & 271/986 (27) & (reference) & & \\
\hline $60-79$ & 433/986 (44) & 2.24 & 1.05 to 4.79 & 0.04 \\
\hline $80+$ & 282/986 (29) & 1.49 & 0.63 to 3.52 & 0.36 \\
\hline \multicolumn{5}{|l|}{ BMI } \\
\hline Underweight & $46 / 810(6)$ & (reference) & & \\
\hline Healthy & $271 / 810$ (33) & 1.5 & 0.34 to 6.76 & 0.58 \\
\hline Overweight & 239/810 (30) & 1.5 & 0.33 to 6.67 & 0.62 \\
\hline Obese & $102 / 810(13)$ & 1.4 & 0.27 to 7.08 & 0.70 \\
\hline Severely obese & $64 / 810(8)$ & 1.1 & 0.17 to 6.75 & 0.93 \\
\hline Morbidly obese & 76/810 (9) & 1.9 & 0.36 to 9.76 & 0.45 \\
\hline Weight & $841 / 986(85)$ & 1.01 & 1.00 to 1.03 & 0.01 \\
\hline Height & 821/986 (83) & 1.00 & 0.99 to 1.00 & 0.55 \\
\hline \multicolumn{5}{|l|}{ Admission diagnosis } \\
\hline Cardiology & 214/986 (22) & (reference) & & \\
\hline Pulmonary & 191/986 (19) & 3.61 & 1.49 to 8.76 & $<0.01$ \\
\hline Oncology/haematology & $125 / 986(13)$ & 2.92 & 1.10 to 7.76 & 0.03 \\
\hline Surgery in past 30 days & $95 / 986(10)$ & 3.15 & 1.14 to 8.74 & 0.03 \\
\hline Other & $355 / 986(36)$ & 0.60 & 0.21 to 1.74 & 0.40 \\
\hline Length of stay & 941/986 (95) & 1.02 & 1.00 to 1.03 & 0.003 \\
\hline Hyperlipidaemia & $231 / 941$ (24.5) & 0.32 & 0.12 to 0.80 & 0.02 \\
\hline Infectious disease & $327 / 986(34)$ & 0.69 & 0.37 to 1.30 & 0.25 \\
\hline Previous VTE & $55 / 986(6)$ & 6.63 & 3.30 to 13.36 & $<0.001$ \\
\hline Surgery within past 30 days & 130/986 (13) & 2.52 & 1.33 to 4.79 & $<0.01$ \\
\hline \multicolumn{5}{|c|}{ Mobility within 1 day of admission: can walk (m) } \\
\hline$>50$ & 187/972 (19) & (reference) & & \\
\hline $10-50$ & 118/972 (12) & 0.50 & 0.16 to 1.59 & 0.24 \\
\hline$<10$ & 336/972 (35) & 0.80 & 0.38 to 1.70 & 0.56 \\
\hline Bedbound & 327/972 (34) & 0.81 & 0.33 to 1.73 & 0.59 \\
\hline Recent fracture & $77 / 986(8)$ & 0.48 & 0.11 to 2.01 & 0.31 \\
\hline \multicolumn{5}{|c|}{ Medication relevant to VTE prophylaxis } \\
\hline None & 206/905 (23) & (reference) & & \\
\hline Anticoagulant* ${ }^{*}$ & 283/905 (31) & 0.50 & 0.42 to 2.08 & 0.87 \\
\hline Other drugs $\dagger$ & 193/905 (21) & 0.80 & 0.15 to 1.33 & 0.15 \\
\hline Anticoagulant other $\ddagger$ & 202/905 (22) & 0.81 & 0.66 to 3.26 & 0.34 \\
\hline \multicolumn{5}{|c|}{ DVT prophylaxis within 48 hours of admission } \\
\hline None & 465 & (reference) & & \\
\hline Prophylactic anticoagulants & 485 & 1.62 & 0.91 to 2.88 & 0.10 \\
\hline
\end{tabular}

was no routine ultrasound scan or test performed to detect asymptomatic VTE occurrence during hospitalisation or following discharge.

\section{Statistical analyses}

Summary descriptive statistics were produced documenting the characteristics of the study population and occurrence of VTE events during the study period. Associations with VTE were examined using univariate logistic regression (for continuous, dichotomous and non-ordinal predictors) and ordinal logistic regression (for ordinal-categorical predictors). Owing to the exploratory nature of the study, a number of risk factors were considered in multiple ways. For example, weight and height were considered as continuous variables and categorical variables, and as continuous and categorical BMI. No statistical correction was made for this multiple testing, so the results should be interpreted as indicative rather than definitive. The development of a multivariate risk model was then undertaken using all variables with a univariate association of $\mathrm{p}<0.25$ and a number of other risk factors deemed to be clinically important. This was done using backwards stepwise multivariate logistic modelling and all first order interaction terms 
were assessed using likelihood ratio tests. The appropriateness of the final multivariate model was assessed using the Hosmer-Lemeshow goodness-of-fit $\left(\chi^{2}(8)\right.$ $=6.93, \mathrm{p}=0.54)$ and the receiver-operating characteristic (ROC) curve (area under the curve $=0.78$ ), indicating that it provided an acceptable fit and level of discrimination (tables 1 and 2). ${ }^{21}$

\section{RESULTS}

A total of 54 patients $(5.5 \%)$ had a diagnosis of VTE during the study period, $40 / 54(74.1 \%)$ of whom were diagnosed during admission while 14/54 (25.9\%) were diagnosed following discharge. The mean age of the patients was 68 years and did not vary by gender (range 17-112, SD 16). The median LOS was 5 days, with an IQR of 2-10 days.

Table 1 presents associations between various potential risk factors and the occurrence of VTE. Gender was not associated with increased VTE risk. Age did not have a significant association with VTE when analysed as a continuous variable (data not shown). However, when analysed as a categorical variable, there was an increased risk of VTE in patients aged above 60 years.

The weight of patients ranged from 38 to $167 \mathrm{~kg}$. Univariate analysis revealed a significant association between weight and the risk of VTE. This was then further analysed in the multivariate model, where for every additional kilogram of weight there was a $1.7 \%$ increase in the likelihood of a VTE, which was statistically significant $(\mathrm{p}=0.01, \mathrm{OR}=1.02,95 \%$ CI 1 to 1.03 ; table 2). Figure 1 indicates that the relationship between weight and VTE is not strictly linear, with risk increasing among those over $120 \mathrm{~kg}$. No association with height or BMI was evident (table 1).

There were 208 patients $(22 \%)$ with an admission diagnosis of cardiology. Of these, 3.3\% had a VTE during the study period (n VTE $=7 / 208$ ). This was used as our reference group when comparing the four other categories of admission diagnoses. In univariate analysis, patients with a pulmonary, oncology/haematology and surgical

Table 2 Association of multivariate risk factors with venous thromboembolism (VTE) in acute medically admitted patients

\begin{tabular}{|c|c|c|c|}
\hline & $\begin{array}{l}\text { Adjusted } \\
\text { OR }\end{array}$ & $95 \% \mathrm{Cl}$ & p Value \\
\hline Previous VTE & 5.83 & 2.17 to 12.99 & $<0.001$ \\
\hline $\begin{array}{l}\text { Surgery within past } \\
30 \text { days }\end{array}$ & 5.02 & 2.13 to 11.79 & $<0.001$ \\
\hline Weight & 1.02 & 1.00 to 1.03 & 0.01 \\
\hline $\begin{array}{l}\text { Infectious disease } \\
\text { Admission diagnosis }\end{array}$ & 0.39 & 0.18 to 0.85 & 0.03 \\
\hline Cardiology & & & 1 \\
\hline Pulmonary & 5.43 & 2.03 to 14.51 & 0.001 \\
\hline $\begin{array}{l}\text { Oncology/ } \\
\text { haematology }\end{array}$ & 2.66 & 0.96 to 7.36 & 0.06 \\
\hline Other & 0.80 & 0.29 to 2.41 & 0.70 \\
\hline
\end{tabular}

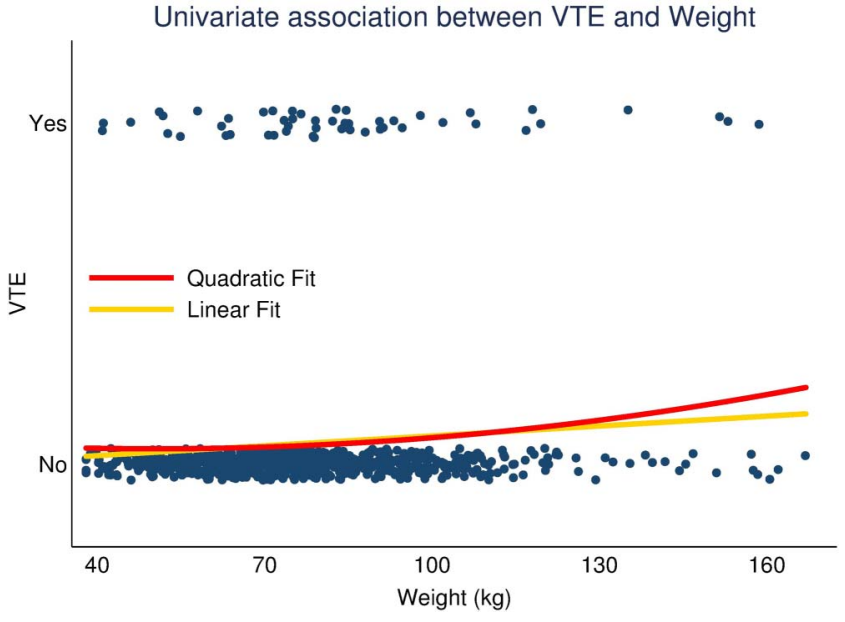

Figure 1 Association of thromboembolic risk and body weight of patients represent in kilogram. VTE, venous thromboembolism.

diagnosis were 3.6, 2.9 and 3.2 times more likely to experience a VTE, respectively. In multivariate analysis, the association between patients with a haematological/ oncological or surgical diagnosis and VTE was no longer significant at the $\alpha=0.05$ level. However, the associations between pulmonary diagnosis and VTE became greater, with these patients being 5.4 times more likely to experience a VTE. Pulmonary diagnoses referred mainly to patients with COPD $(87 / 179)$ and pneumonia $(77 / 179)$, in addition to pneumothorax, pleural effusion and interstitial pulmonary oedema (15/179).

Although there was a univariate association between LOS and VTE (OR 1.02, $\mathrm{p}=0.003$ ), based on the assumption that LOS is not a risk factor for short-stay patients, a subsequent analysis found an association when LOS was $>7$ days $(\mathrm{p}=0.046, \quad \mathrm{OR}=1.01,95 \%$ CI 1.00 to 1.03$)$; however, this association was not sustained during multivariate modelling.

Previous VTE was associated with an increased risk of developing VTE (table 1). This association remained significant in multivariate analysis, with these patients being 5.8 times more likely to develop a VTE (table 2).

In our cohort, 130/941patients (13\%) had a history of surgery within the past 30 days. Patients who had a surgery within the past 30 days were five times more likely to have a VTE during the study period than those who did not $(\mathrm{p}<0.001, \mathrm{OR}=5.02,95 \%$ CI 2.14 to 11.79$)$. Gender appeared to affect the rate at which patients who had recent surgery experienced a VTE. Of the 14 patients with VTE in the context of recent surgery, 10 $(71 \%)$ were male, and $4(29 \%)$ were female. However, this difference was not statistically significant $\left(\chi^{2}(1)\right.$ $=1.58, \mathrm{p}=0.21)$. Nevertheless, given the very small numbers involved and the exploratory nature of the analysis, this may represent an interesting finding.

Dyslipidaemia, while it was not included in the multivariate analysis, was found to have a significant association with decreased risk of VTE. 
Of the $33 \%$ of patients admitted with an acute infection, $4.5 \%$ had a VTE during the study period (n VTE $=14 / 309$ ), compared with $6.4 \%$ of patients who did not have an acute infection (n VTE $=40 / 625)$. This was statistically significant but a small decrease in VTE risk and may not have clinical relevance as this was overlapping with other diagnoses $(\mathrm{p}=0.02, \mathrm{OR}=0.40,95 \%$ CI 0.19 to $0.84, \mathrm{n}=14 / 295)$.

Mobility within 24 hours of admission and recent fractures were not associated with VTE.

Other negative findings of interest include a lack of any association between the use of medications relevant to VTE or anticoagulants specific for thromboprophylaxis and the occurrence of VTE.

Overall $74 \%$ of patients were taking some form of anticoagulants, including enoxaparin, aspirin, clopidogrel, low-dose heparin and other non-steroidal antiinflammatory drugs (NSAIDs). VTE-specific prophylaxis with enoxaparin or equivalent (at the standard dose for the institution with $40 \mathrm{mg}$ daily until discharge) was recorded in $53 \%$ of participants (table 1). Some form of anticoagulation including antiplatelet agents were used in $63 \%$ of patients who developed VTE.

Potential risk factors such as hypertension, diabetes, eGFR/creatinine clearance and heart failure did not show statistically significant associations with VTE.

\section{DISCUSSION}

This prospective cohort study examined the incidence of and risk factors for VTE in acute medical patients admitted to a medium-sized hospital during the period of hospitalisation and for 3 months following discharge. This study contributes additional knowledge to our understanding of VTE in medical patients and highlights the fact that a significant proportion of VTE events occur during admission and in the period following discharge. This could help future development of strategies to identify medical patients who are at sufficiently high risk to warrant an extended thromboprophylaxis.

This study identified several factors associated with an increased risk of VTE. Some, such as previous VTE and recent surgery, are already well established while other factors identified in our study, such as pulmonary diagnoses and increased body weight, could provide new insights into clinical risk stratification.

The increased risk conferred by a history of VTE and recent surgery agrees with results from a number of other studies. ${ }^{15} 16$ Our finding of pulmonary diagnoses, as defined above, increasing the risk of VTE by five times compared with other admission diagnoses, is worth further investigation. Other studies have shown conditions such as COPD, respiratory failure and acute or chronic chest infection to be independent risk factors ${ }^{22-}$ ${ }^{24}$ and inclusion of such diagnoses may help explain our finding of such a large increase in risk associated with a pulmonary admission diagnosis. A recent epidemiological study on 8646 patients showed that patients with stage III/IV COPD had a twofold higher risk of secondary VTE compared with patients with normal airflow (HR 2.05, 95\% CI 1.02 to 4.10). ${ }^{25}$ However, it is worth noting that the studied cohort was non-hospitalised patients. A possible explanation could be that pulmonary conditions are generally associated with a certain degree of inflammation and cytokine release. This inflammatory component may confer an increased risk for VTE.

Patients admitted with a cardiology diagnosis (used as a reference group to compare with the other admission diagnosis categories) had a lower incidence of VTE. One possible explanation for this observation is that many cardiology patients were receiving a concomitant antiplatelet therapy. However, the association between cardiology admission diagnosis, medications and VTE was not assessed. Overall, patients who were started on NSAIDs or antiplatelet medications did not show a statistical difference with the patients with VTE who were not on such medications.

Further possible risk factors suggested by this study but which were not statistically significant were male gender in patients with recent surgery, increasing body weight and oncology/haematology diagnosis. While we did not demonstrate a statistically significant difference in the incidence of VTE in these groups, the number of patients with VTE was small and our study was exploratory in nature. These possible associations may prove fertile should they be considered in larger, more focused studies.

Interestingly, increasing weight had a statistically significant association with VTE, but no statistical significance was found with BMI. This is in conflict with emerging evidence suggesting obesity as a potential risk factor for VTE. ${ }^{26}$ The small number of VTEs within BMI categories partly accounts for this. BMI is also a simplistic measure of body composition that is inferior to other more sensitive measures such as dual-energy X-ray absorptiometry (DXA) measurements. ${ }^{28}{ }^{29}$ Future studies should consider further exploration of the interplay between BMI and other potential confounders such as immobility and metabolic syndrome-associated inflammation. ${ }^{30}$

We did find a slight protective association between infection diagnosis and risk of VTE. In this study those patients with a diagnosis of acute infection had less than half the risk of developing VTE, compared with other patients. This is in conflict with the findings in previous studies. ${ }^{31}$ However, our observation may have been affected by the relatively low numbers.

One of the strengths of this study is that it included follow-up of patients 3 months postdischarge. In this study, $26 \%$ of patients with VTE developed or were diagnosed with VTE after discharge from hospital (14/54). This finding, together with the observation of associated risk factors has two important implications. First, it is of particular relevance to clinicians assessing patients in the outpatient setting. When assessing patients in the community with possible VTE-related symptoms, this 
study suggests that we should consider both patients with already well-established risk factors such as recent surgery, previous VTE and malignancy, and any recent admissions for medical conditions, especially with pulmonary diagnoses. Such consideration could significantly change the rate of successful VTE prevention and treatment and hence patient outcomes. The role that other factors such as age, gender and BMI should play in clinical assessment requires further study.

Second, the extended follow-up postdischarge highlights the fact that a significant number of VTE events occur in the period following discharge from hospital. There are only a limited number of studies examining the incidence of VTE in the postdischarge period, but they have revealed similar findings. ${ }^{32}$ The question has therefore been asked whether thrombosis prophylaxis should be continued beyond the standard initial course. The benefit of extended duration prophylaxis for certain types of surgical patients has been established in RCTs and the practice generally accepted. ${ }^{20}$ However, there are very limited studies of the same issue for medical patients. Only one RCT of extended duration thrombosis prophylaxis in acute medical patients with reduced mobility has been published. ${ }^{33}$ This study demonstrated a reduction of six symptomatic DVTs per 1000 and 1 less fatal PE, but at the expense of 5 more major bleeding events per 1000 and no change in overall mortality. While the American College of Chest Physicians (ACCP) concluded in 2012 that extended duration prophylaxis for at-risk medical patients was not recommended, the issue remains controversial and further investigation is warranted. ${ }^{33}{ }^{34}$ We believe that a large multicentre international trial has recently started to address such important questions and we look forward to seeing the outcome of this research (https:// clinicaltrials.gov/ct2/show/NCT02111564).

In our study, $63 \%$ of patients who developed VTE were receiving some form of thromboprophylaxis in hospital compared with $37 \%$ of patients with VTE who were not (data were missing for two patients). It is important to note that the hospital in which this study was conducted has a current policy that all acute medical inpatients, regardless of type of diagnosis or other individual risk factors, should receive thromboprophylaxis provided there are no contraindications. This study was observational and exploratory, and its purpose was not to assess the effectiveness of or adherence to current thromboprophylaxis regimens. However, it seems likely that a significant number of patients are not receiving prophylaxis in accordance with such a guideline, especially given the poor compliance with prophylaxis guidelines for medical patients found in large multinational studies. ${ }^{18}$ Further research should be conducted specifically looking at adherence to local prophylaxis guidelines in this centre and whether such guidelines should be modified to include all high-risk patients (such as a pulmonary admission diagnosis) while at the same time avoid unnecessary prophylaxis of low-risk patients. Development and implementation of a risk assessment tool applicable to the local environment could improve overall compliance with and effectiveness of prophylaxis strategies.

Refinement of guidelines for VTE prophylaxis in medical patients and improvements in compliance with these guidelines could have significant economic benefits to the healthcare system, particularly in the light of findings, such as an Italian study ${ }^{35}$ that demonstrated four times greater cost in VTE management as compared with VTE prophylaxis, together with the substantial actual value cost of VTE events reported in a number of other studies. ${ }^{8-12}$ While we should be careful in extrapolating such figures to our own local context, with similar findings reported in a number of countries, the value of VTE prevention is reinforced.

Limitations of our study include the fact that it was conducted in a single centre over a short duration with a relatively small number of patients. As this was an observational, non-interventional study, there was no test performed routinely for the purposes of the study to detect asymptomatic VTE, during or after discharge. Some cases with asymptomatic VTE, therefore, may have been missed. Furthermore, incomplete data included the number of missing patients who were taking medications relevant to VTE or missing admission diagnosis may affect the power of the study.

In summary, this study adds to the evidence base and our understanding of VTE in medical patients. By extending the follow-up of patients for 3 months after the hospitalisation period, it highlights the fact that a significant number of patients develop VTE after discharge from hospital. Thus, it reinforces the need for further investigation into postdischarge VTE, including identification of high-risk patients and appropriate thromboprophylaxis strategies, and highlights the importance of involving the primary care sector in the assessment and management of these patients.

Twitter Follow Terry Hannan at @tjhannan

Acknowledgements The authors wish to acknowledge and thank Rebecca Li, Anurag Arora, Imal Hemachandra, Michael Besley, Quan Tran and Richard Woodford from School of Medicine, University of Tasmania who contributed in recruiting the patients and provided technical support with electronic data capture forms. The authors also wish to sincerely thank Professor Dominic Geraghty, Head of School of Health Sciences, University of Tasmania, Tasmania, Australia, for his kind help and technical support in providing the University facilities for the authors to enable conduction of the study. Also they sincerely thank Mary Sexton and Laurie McEwen from the Haematology Research Unit, Pathology Department, Launceston General Hospital, Australia, for their enormous help and technical support.

Contributors AAK is the principal investigator of the study who designed the trial and organised and coordinated all aspects of the research including all steps of the manuscript preparation. He is responsible for the study concept, design, writing, reviewing, editing and approving the manuscript in its final form. BEK, SW, YCF, NR, JL, RM and CMC recruited the patients and contributed in the study design, analysis and interpretation of data, writing the manuscript and reviewed and approved the manuscript. EM conducted the statistical analyses and analysed the data, drafted the results section and finally approved the manuscript. CS and TH recruited the patients, supervised 
the medical wards at the Launceston General Hospital and drafted and finally approved the manuscript.

Funding This study was supported by the Pathology Department, Launceston General Hospital, Launceston, Tasmania, Australia in affiliation to the University of Tasmania, Australia. Pathology Department provided logistic and monetary support. This is an investigator-initiated study.

Disclaimer All aspects of the study conception, design, administration, data collection, analysis and presentation have been undertaken independently of any sponsor. The authors declared no conflicts of interest.

Competing interests None declared.

Patient consent Obtained.

Ethics approval Tasmanian Human Research Ethics Committee, Australia.

Provenance and peer review Not commissioned; externally peer reviewed.

Data sharing statement No additional data are available.

Open Access This is an Open Access article distributed in accordance with the Creative Commons Attribution Non Commercial (CC BY-NC 4.0) license, which permits others to distribute, remix, adapt, build upon this work noncommercially, and license their derivative works on different terms, provided the original work is properly cited and the use is non-commercial. See: http:// creativecommons.org/licenses/by-nc/4.0/

\section{REFERENCES}

1. Hobbs M, Ridout S, Stewart L, et al. The incidence and risk factors for venous thromboembolism in hospitals in Western Australia 19992001. Melbourne: The School of Population Health, University of Western Australia, 2005.

2. Heit JA, Melton LJ III, Lohse CM, et al. Incidence of venous thromboembolism in hospitalized patients vs community residents. Mayo Clin Proc 2001;26:1102-10.

3. Beckman MG, Hooper WC, Critchley SE, et al. Venous thromboembolism: a public health concern. Am J Prev Med 2010;38 (4 Suppl):S495-501.

4. Baglin TP, White $\mathrm{K}$, Charles A. Fatal pulmonary embolism in hospitalised medical patients. J Clin Pathol 1997;50:609-10.

5. Clinical practice guideline for the prevention of venous thromboembolism (deep vein thrombosis and pulmonary embolism) in patients admitted to Australian hospitals. N.H.a.M.R. Melbourne, 2009.

6. Centers for Disease Control and Prevention (CDC). Venous thromboembolism in adult hospitalizations-United States, 20072009. MMWR Morb Mortal Wkly Rep 2012;61:401-4.

7. Heit JA. Venous thromboembolism: disease burden, outcomes and risk factors. J Thromb Haemost 2005;3:1611-17.

8. Bullano MF, Willey $\mathrm{V}$, Hauch $\mathrm{O}$, et al. Longitudinal evaluation of health plan cost per venous thromboembolism or bleed event in patients with a prior venous thromboembolism event during hospitalization. J Manag Care Pharm 2005;11:663-73.

9. Hawkins D. Economic considerations in the prevention and treatment of venous thromboembolism. Am J Health Syst Pharm 2004;61(Suppl 7):S18-21.

10. Deitelzweig SB, Becker R, Lin J, et al. Comparison of the two-yea outcomes and costs of prophylaxis in medical patients at risk of venous thromboembolism. Thromb Haemost 2008;100:810-20.

11. Dobesh PP. Economic burden of venous thromboembolism in hospitalized patients. Pharmacotherapy 2009;29:943-52.

12. Ashrani AA, Heit JA. Incidence and cost burden of post-thrombotic syndrome. J Thromb Thrombolysis 2009;28:465-76.

13. Anderson FA Jr, Wheeler HB. Venous thromboembolism. Risk factors and prophylaxis. Clin Chest Med 1995;16:235-51.

14. Wickham N, Gallus AS, Walters BNJ, et al. Prevention of venous thromboembolism in patients admitted to Australian hospitals: summary of National Health and Medical Research Council clinical practice guideline. Intern Med J 2012;42:698-708.

15. Heit JA, Silverstein MD, Mohr DN, et al. Risk factors for deep vein thrombosis and pulmonary embolism: a population-based case-control study. Arch Intern Med 2000;160:809-15.

16. Alikhan R, Cohen AT, Combe S, et al., MEDENOX Study. Risk factors for venous thromboembolism in hospitalized patients with acute medical illness: analysis of the MEDENOX Study. Arch Intern Med 2004;164:963-8.

17. Anderson FA Jr, Spencer FA. Four topics in venous thromboembolism risk factors for venous thromboembolism. Circulation 2003;107:19-16.

18. Cohen AT, Tapson VF, Bergmann JF, et al. Venous thromboembolism risk and prophylaxis in the acute hospital care setting (ENDORSE study); a multinational cross-sectional study. Lancet 2008;371:387-94.

19. National Health and Medical Research Council. Preventing venous thromboembolism in hospitalised patients: summary of NHMRC activity 2003-2010. Melbourne: National Health and Medical Research Council, 2011.

20. Spyropoulos AC, Anderson FA, FitzGerald G, et al., IMPROVE investigators. Predictive and associative models to identify hospitalized medical patients at risk for VTE. Chest 2011;140:706-14.

21. Hosmer DW, Lemeshow S. Applied logistic regression. 2nd edn. New York: John Wiley \& Sons. 2000:147-63.

22. Rizkallah J, Man SP, Sin DD. Prevalence of pulmonary embolism in acute exacerbations of COPD: a systematic review and metaanalysis. Chest 2009;135:786-93.

23. Gunen $\mathrm{H}$, Gulbas $\mathrm{G}$, In E, et al. Venous thromboemboli and exacerbations of COPD. Eur Respir J 2010;35:1243-8.

24. Anderson FA, Spencer FA. Risk factors for venous thromboembolism. Circulation 2003;107(Suppl 1):I-9-16.

25. Børvik T, Brækkan S, Enga K, et al. COPD and risk of venous thromboembolism and mortality in a general population. Eur Respir $J$ 2016;47:473-81.

26. Stein PD, Beemath $\mathrm{A}$, Olson RE. Obesity as a risk factor in venous thromboembolism. Am J Med 2005;118:978-80.

27. Klovaite J, Benn M, Nordestgaard BG. Obesity as a causal risk factor for deep venous thrombosis: a Mendelian randomization study. J Intern Med 2015;277:573-84.

28. Foong YC, Chherawala N, Aitken D, et al. Accelerometerdetermined physical activity, muscle mass, and leg strength in community-dwelling older adults. J Cachexia Sarcopenia Muscle 2016;7:275-83.

29. Foong YC, Aitken D, Winzenberg T, et al. The association between physical activity and reduced body fat lessens with age-results from a cross-sectional study in community-dwelling older adults. Exp Gerontol 2014:55:107-12.

30. Ay C, Tengler T, Vormittag R, et al. Venous thromboembolism-a manifestation of the metabolic syndrome. Haematologica 2007;92:374-80.

31. Barba R, Zapatero A, Losa JE, et al. Venous thromboembolism in acutely ill hospitalised medical patients. Thromb Res 2010;126:276-9.

32. Kahn SR, Lim W, Dunn AS, et al. American College of Chest Physicians. Prevention of VTE in nonsurgical patients: antithrombotic therapy and prevention of thrombosis: American College of Chest Physicians evidence-based clinical practice guidelines. Chest 2012;141(2 Suppl):e195S-226S.

33. Hull RD, Schellong SM, Tapson VF, et al. EXCLAIM (Extended Prophylaxis for Venous ThromboEmbolism in Acutely III medical Patients With Prolonged Immobilization) study. Extended-duration venous thromboembolism prophylaxis in acutely ill medical patients with recently reduced mobility: a randomized trial. Ann Intern Med 2010;153:8-18.

34. Sharma A, Chatterjee S, Lichstein E, et al. Extended thromboprophylaxis for medically ill patients with decreased mobility does it improve outcomes? J Thromb Haemost 2012;10:2053-60.

35. Gussoni G, Foglia E, Frasson S. et al. Real-world economic burden of venous thromboembolism and antithrombotic prophylaxis in medical inpatients. Thromb Res 2003;131:17-23. 